\title{
The sensitivity and specificity of fecal and cecal culture for the detection of Campylobacter in Dutch broiler flocks quantified by Bayesian analysis
}

\author{
E. Woldemariam, A. Bouma*, J.C.M. Vernooij, A. Stegeman \\ Department of Farm Animal Health, Faculty of Veterinary Medicine, Utrecht University, The Netherlands
}

Received 24 May 2007; received in revised form 11 October 2007; accepted 6 November 2007

\begin{abstract}
Dutch broiler flocks are routinely tested for the presence of thermotolerant Campylobacter spp. using a standard cultural procedure for fecal and cecal samples. The objective of this study was to estimate the sensitivity and specificity of fecal and cecal culture for detection of Campylobacter colonization in broiler flocks in absence of a gold standard. Data from 1600 flocks were used from two different populations, whereby only flocks with both fecal and cecal culture results were included in the analysis. Latent class analysis using Bayesian inference was applied to generate the test characteristics of fecal and cecal culture. Two statistical models assuming conditional dependence of both tests on Campylobacter status were used to compare the results. On flock level, the sensitivity of the fecal culture was found to be $21 \%$ (95\% CI: 12 , $31)$ and $23 \%(95 \%$ CI: 13, 60), and the specificity was 98\% (95\% CI: 94, 99) and 97\% (95\% CI: 92, 99) for the two models, respectively. The sensitivity of the cecal culture was 64\% (95\% CI: 37, 89) and 66\% (95\% CI: 39, 90), and the specificity was 98\% (95 CI: 94, 99) and 95\% (95\% CI: 72, 99) in respective models. The implications of a low sensitivity as in the case of the fecal culture is important for the design and interpretation of monitoring programmes and may result in excessive false negative test results. Although cecal culture is the more sensitive test, substantial misclassification of infected flocks may still occur.
\end{abstract}

(C) 2007 Elsevier B.V. All rights reserved.

Keywords: Campylobacter; Cecal culture; Fecal culture; Sensitivity; Specificity; Bayesian inference

\section{Introduction}

Nowadays thermotolerant Campylobacter species, C. jejuni and $C$. coli, are the most common cause of acute bacterial gastroenteritis in humans in most developed countries (Wingstrand et al., 2006; Skirrow, 1991; Havelaar, 2004) and the incidence of Campylobacter infection has been increasing in many industrialized countries (Lund et al., 2004; Engberg et al., 2001; De Wit et al., 2001). Although rarely fatal, Campylobacter infections cause considerable illness and loss of productivity and may be associated with severe disabling consequences, including reactive arthritis and Guillain-Barré Syndrome (Wingstrand et al., 2006; Altekruse et al., 1999).

Thermotolerant Campylobacter species live in a wide range of animals, but various studies have shown that poultry and

\footnotetext{
* Corresponding author. Marburglaan 2, 3584 CN Utrecht, The Netherlands. Tel.: +31 30 2531013; fax: +31302521887.

E-mail address: a.bouma@uu.nl (A. Bouma).
}

poultry products are the main reservoirs of Campylobacter spp. for human infection mainly C. jejuni (Jacobs-Reitsma, 1997; Newell and Fearnley, 2003). Several control measures have been implemented to reduce the exposure of humans to Campylobacter spp., either by reducing the incidence of Campylobacter infections in broiler flocks by biosecurity measures at farms or by improving slaughterhouse hygiene (e.g. Wagenaar et al., 2006). Although proper hygienic measures may reduce the incidence of infections of poultry flocks, it is yet by far not a guarantee that the flock will remain free from Campylobacter spp. (Adkin et al., 2006), and consequently cannot rely on as a sole intervention to minimize exposure to humans.

Improvement of intervention strategies that reduce human exposure requires knowledge of the Campylobacter status of broiler flocks. Consequently, it is essential to have data on the sensitivity and specificity of tests to detect an infection at flock level. Generally flocks are tested for the presence of Campylobacter by culturing fecal and/or cecal samples (Corry et al., 1995; Musgrove et al., 2001; Payne et al., 1999; Berndston 
et al., 1996). Fecal samples are easier to gather, but it is generally assumed that cecal samples are more appropriate, since Campylobacter mainly colonizes the cecum and, consequently, fecal samples often contain a lower number of bacteria per gram than cecal samples (Rudi et al., 2004).

The sensitivity and specificity of the two testing procedures have, to our knowledge, never been established. This quantitative information is essential to interpret test results which is, in turn, necessary to develop intervention measures to further reduce human exposure. Preferably, test characteristics are determined by comparing the results with those from a reference test, but perfect reference tests are not available. In absence of the true infection status of animals a Bayesian approach of maximizing the likelihood for the model can be performed (Enøe et al., 2001, Frossling et al., 2003, Orr et al., 2003, Swildens et al., 2005). In this approach, it is assumed that every valid test (a test that performs better than chance) classifies a proportion of the true positive and true negative testsamples correctly (Casella and George, 1992). Moreover, in the Bayesian approach to estimate a parameter, e.g. sensitivity Se, prior information about this parameter Se (from the literature or expert opinion) is used in combination with actual data to obtain a posterior estimate of Se (Branscum et al., 2005).

The aim of this study was to estimate and compare the sensitivity and specificity of fecal and cecal culture for detection of Campylobacter species in Dutch broiler flocks using a Bayesian analysis.

\section{Materials and methods}

\subsection{Study population}

Since 1997, a monitoring programme for Campylobacter colonization of Dutch broiler farms is carried out by The Dutch Product Board of Livestock and Meat (PVE). In this study we used data from 1600 flocks. Out of these 1600 flocks 579 had both fecal and cecal culture results, and belong either to the partial and/or final depletion population (Table 1). About 8\% (47) of the flocks have undergone both partial and final depletion. Partial depletion is a process where a small part of early market-weight attaining broilers are slaughtered, followed by final depletion of the remaining flock, usually one week later (Jacobs-Reitsma et al., 2001). Because of the economic benefit, it is practiced by several farmers and slaughter companies. An overview of the technical data is given in Table 2.

Table 1

Observed test results of fecal and cecal Campylobacter culture cross-classified into partial and final depletion population

\begin{tabular}{|c|c|c|c|c|c|c|c|}
\hline & & Cecal cu & Iture & & & & \\
\hline & & Partial & & & Final & & \\
\hline & & Positive & Negative & Total & Positive & Negative & Tota \\
\hline Fecal culture & Positive & 12 & 5 & 17 & 30 & 18 & 48 \\
\hline & Negative & 27 & 57 & 84 & 119 & 311 & 430 \\
\hline & Total & 39 & 62 & 101 & 149 & 329 & 478 \\
\hline
\end{tabular}

Table 2

An overview of the technical data of the Dutch broiler flocks tested for Campylobacter

\begin{tabular}{lll}
\hline & Partial depletion & Final depletion \\
\hline No flocks & 101 & 478 \\
$\begin{array}{l}\text { Mean no. chicken per flock } \\
\text { Age (min, max)* }\end{array}$ & 80,269 & 78,227 \\
$\begin{array}{l}\text { Percent positives** } \\
\text { Spring }\end{array}$ & $35(34,39)$ & $40(34,50)$ \\
Summer & $48(14 / 29)$ & \\
Fall & $70(19 / 27)$ & $6(9 / 134)$ \\
$\quad$ Winter & $20(4 / 20)$ & $6(7 / 107)$ \\
\hline
\end{tabular}

*Age in days at slaughter.

**Considering a flock positive when the fecal and/or the cecal culture tested positive.

\subsection{Collection and processing of samples}

Fecal samples ( 5 per shed) were collected by the farmer a few days before slaughter, put in a sampling bag or tube with identification, and sent to a certified laboratory which was approved by the PVE. The samples were sent by regular mail, generally within 1-2 days after sampling. Cecal samples (30 per batch) were collected at slaughterhouses, and sent to the laboratory immediately. Within $48 \mathrm{~h}$ after arrival in the laboratory, samples were examined for the presence of Campylobacter by laboratories certified by the PVE (ISO/IEC 17025; www.pve.nl). This includes direct streaking of sampled material on to a Campylobacter selective mCCDA plate, followed by micro-aerobic incubation at $41.5{ }^{\circ} \mathrm{C}$ for $48 \mathrm{~h}$. According to the PVE protocol, specific colonies are confirmed by oxidase reaction and microscopically. In addition, some samples may be confirmed serologically or by agglutination test (ISO $10272: 1995 /$ Cor 1997). The test results were registered in a database at the slaughterhouse.

\subsection{Statistical analysis}

The values of Se and $\mathrm{Sp}$ of the two testing procedures were estimated by Bayesian analysis using the winBUGS program (Spiegelhalter et al., 1996). The test results of fecal and cecal culture may not be independent as they are based on the same biological phenomenon being presence of Campylobacter (Gardner et al., 2000). When using a model assuming independence of data, estimates of test accuracy could be misleading if the test outcomes for a given animal appeared to be correlated (Georgiadis et al., 2003), which would result in a biased estimate. Therefore, a model assuming conditional dependence on infection status was used. Such a model would make clear if the data were independent or not.

Two models were used that assume conditional dependence on infection status between tests (Engel et al., 2006 (model 1) and Branscum et al., 2005 (model 2)). The models allow the estimation of the sensitivity and specificity of two tests, based on their cross-classified results, when applied to flocks from two populations with different disease prevalences (Enøe et al., 2001). These parameters have a distribution in stead of a fixed value. The distributions are used to find a combined optimum 
for the sensitivity and specificity and the prevalences in that the likelihood for the model is maximal given the data. The parameterization of both models is different. The model of Engel et al. (2006) is based on a normal distribution of the priors, whereas the model of Branscum is based on beta distributions. Posterior distributions were obtained with Markov Chain Monte Carlo (MCMC) methods employing the Gibbs sampler, as implemented in the WinBUGS program.

For the analysis, two populations with a different prevalence of infection had to be made from the data set: one being partially depleted flocks and the second being the finally depleted flocks. Therefore the samples were grouped into those originating from the partial and those originating from final depletion flocks (Table 1). For each population test results are cross-classified in a $2 \times 2$ table according to the status of each flock tested (as shown in Table 1). Each $2 \times 2$ table provides three degrees of freedom for estimation. When two populations are available, there are $6 \mathrm{~d}$. f. for estimation. The number of parameters of interest is two for each of the two tests (sensitivities and specificities) and one for each of the two populations (prevalences).

The priors of both test procedures indicate that we assumed that the sensitivity of both tests likely would be in excess of $10 \%(\operatorname{dbeta}(1.53,1.53))$ and that the specificity of both fecal and cecal culture was high, i.e. $95 \%$ sure that it is greater than $95 \%$ (dbeta $(88.279,1.88)$ ) (Wagenaar, pers. comm.). The latter was because growth of bacteria in the selective media has a very low probability of resulting in a positive result. The prior information of the sensitivity and specificity of fecal and cecal culture is shown in Table 3. The prior for the true prevalence of Campylobacter in the partially depleted and the finally depleted population was set to an uninformative uniform beta distribution $(\operatorname{dbeta}(1,1))$ (see also Table 3 ). For the analyses presented, posterior inferences were based on 50,000 iterations after a burn-in of 5000 iterations being discarded. Convergence was assessed by observing the autocorrelation and by running multiple chains from dispersed starting values and investigating the Brooks-Gelman-Rubin convergence statistic (Gelman and Rubin, 1992). The median of the posterior distribution was used as an estimate for our parameter of interest. The 2.5 and 97.5 percentage points were used for estimating the $95 \%$ credibility intervals.

Sensitivity analysis was done by doing separate analyses of the partially and finally depleted population to check if the test characteristics varied across the two populations. In addition, repeated analysis was done for different initial values and priors to see the stability of the output.

\section{Results}

\subsection{Descriptive statistics}

\subsubsection{Partially depleted population}

The total number of flocks in the partially depleted population was 101, and the apparent prevalence of Campylobacter in this population based on either fecal and/ or cecal culture positive was $44 \%$. There were 17 (16\%) flocks with a positive fecal culture and $39(38 \%)$ flocks with a positive cecal culture (Table 1$)$. The mean age at slaughter of the partial depleted flocks was 35 days, which ranges between 34 and 39 days (Table 2).

\subsubsection{Finally depleted population}

There were 478 finally depleted flocks, out of which 47 flocks also had undertaken partial depletion. The apparent prevalence of Campylobacter considering positive fecal and/or cecal culture as a colonized flock was $35 \%$. There were 48 (10\%) flocks tested positive with the fecal culture and 139 (29\%) flocks positive with cecal culture (Table 1). The mean age at slaughter was 40 days, which ranges between 34 and 50 days (Table 2 ).

\subsection{Sensitivity and specificity}

Model 1 resulted in a sensitivity of $23 \%(95 \%$ CI 13, 60) and a specificity of $97 \%(95 \%$ CI: 92,99$)$ of the fecal test and a sensitivity of $66 \%(95 \%$ CI 40,90$)$ and a specificity of $96 \%$ (95\% CI: 72, 99) of the cecal test (Table 4). The results of model 2 were very similar, a sensitivity of $21 \%(95 \%$ CI 12,32$)$ and a specificity of $98 \%(95 \% \mathrm{CI}: 94,99)$ of the fecal test and a sensitivity of $64 \%(95 \%$ CI 37,89$)$ and a specificity of $98 \%$ (95\%CI: 94, 99) of the cecal test, respectively. Model 1 indicated a true prevalence $60 \%(95 \% \mathrm{CI}: 37,96)$ and $46 \%$ (95\% CI: 31, 83) for the partial and final depletion population, while model 2 indicated a partial and final depletion population true prevalence of 54\% (95\% CI: 21,91$)$ and 40\% (95\% CI: 11 , $72)$, respectively. The correlation for the sensitivity of the two tests was $0.02(95 \% \mathrm{CI}:-0.4,0.24)$ and for the specificity 0.45

Table 3

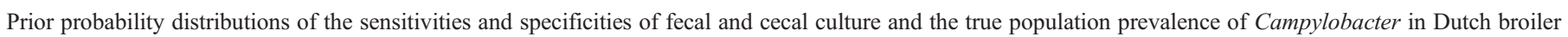

\begin{tabular}{|c|c|c|c|c|c|}
\hline Parameter & & Beta distribution & $\begin{array}{l}\text { Mode } \% \\
\text { ( } 95 \% \text { lower bound) }\end{array}$ & Source & Convergence \\
\hline \multirow[t]{2}{*}{ Fecal culture } & Sensitivity & $\operatorname{dbeta}(1.53,1.53)$ & $50(10)$ & $\begin{array}{l}\text { It is very low usually with mode } 50 \% \\
\text { (Wagenaar pers. Comm..) }\end{array}$ & Converged \\
\hline & Specificity & $\operatorname{dbeta}(88.279,1.88)$ & $99(95)$ & $\begin{array}{l}\text { It is unlikely that there would be false positives } \\
\text { (Wagenaar pers. Comm..) }\end{array}$ & Converged \\
\hline \multirow{2}{*}{ Cecal culture } & Sensitivity & $\operatorname{dbeta}(1.53,1.53)$ & $50(10)$ & Same as Se fecal culture & Converged \\
\hline & Specificity & dbeta(88.279,1.88) & $99(95)$ & Same as Sp fecal culture & Converged \\
\hline \multirow[t]{2}{*}{ True prevalence } & Partial depletion & $\operatorname{dbeta}(1,1)$ & & Uninformative & Less converged ${ }^{* *}$ \\
\hline & Final depletion & $\operatorname{dbeta}(1,1)$ & & Uninformative & Less converged $* *$ \\
\hline
\end{tabular}

*Convergence was assed by observing the Gelman Rubin statistic and Autocorrelation.

**Less convergence of the two prevalence parameters may be attributed to the uninformative nature of the priors. 
Table 4

Estimates and 95\% credibility intervals of sensitivity and specificity of fecal and cecal culture and the true prevalence of Campylobacter in Dutch broiler flocks estimated by Bayesian inference using two models assuming conditional dependence

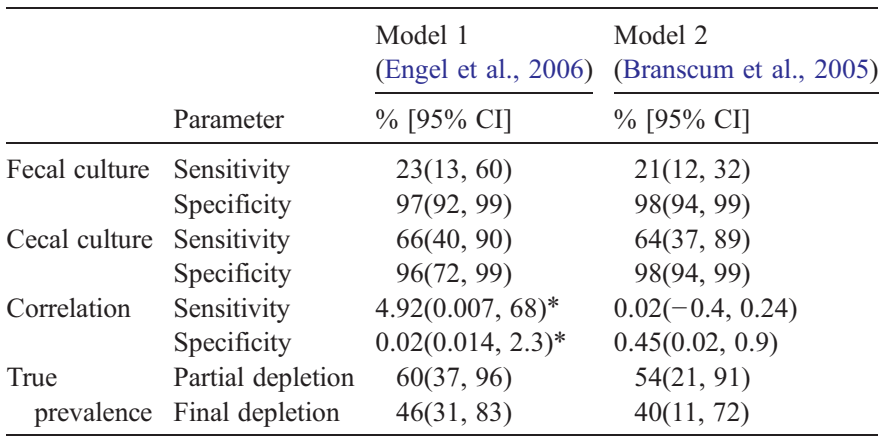

*Percentage increase.

(95\% CI: 0.02, 0.9). This means that the tests behave like independent tests for the parameter sensitivity and are slightly correlated with respect to the parameter specificity.

The sensitivity and the specificity remained consistent for both analyses on a partial or final depletion population level, and on a combined level verifying the assumption that test characteristics be the same across the two populations. Further sensitivity analysis was done to see how the priors and initial values influenced the final output. According to expert opinion the sensitivity of Campylobacter culture is generally low, and resulted in a prior of $50 \%$ (with a $95 \%$ interval from $10-90 \%$ ). To determine a possible effect of a change in estimate, the priors were changed from a mode of $50 \%$ and $95 \%$ lower limit of $10 \%$ $\operatorname{dbeta}(1.53,1.53)$ to a mode of $50 \%$ and $95 \%$ lower limit of $30 \%$ $(\operatorname{dbeta}(8.00,8.00))$. This implies that not only the lower limit is increased to $30 \%$ but also that the upper limit is decreased to $70 \%$. This change of priors did not result in a different outcome.

\section{Discussion}

The aim of this study was to quantify the sensitivity and specificity of the whole testing procedure including fecal and cecal culture for detection of Campylobacter colonization of broiler flocks. Field data were used and two Bayesian models, which assume conditional dependence on infection status, were applied to analyse the data. Both models resulted in comparable estimates for all parameters. In both models it appeared that the sensitivity of cecal culture was much higher than the sensitivity of the fecal culture. The implication of this finding is that it can now be determined how many flocks may have been misclassified as negative. This, in turn, could mean that human exposure to Campylobacter might have been much higher than assumed until now. The results can also be used to determine whether and how the test procedure and herd sensitivity could be improved.

The lower sensitivity of the fecal compared to cecal culture could be explained by previous findings that Campylobacter mainly colonizes the ceca (Rudi et al., 2004). Another explanation is that fecal samples were collected by the farmer and the interval between sample collection and laboratory testing, and the possible mishandling might have exposed the bacterium to sub-optimal conditions resulting in less culturable bacteria (Havaei et al., 2006). In addition to this, fewer fecal samples are taken than cecal samples, which in general also decreases herd sensitivity (e.g. Dohoo et al., 2003). Concerning the specificity, both the cecal and fecal culture showed high values, because growth of Campylobacter in selective culture media is usually considered as unambiguous demonstration of infection.

Sensitivity analysis on partially or finally depleted population and combined population level was done to verify the validity of the assumption that test characteristics were comparable between the two populations. The results showed that the sensitivity and the specificity remained within the same credibility interval. The low correlation between the sensitivity of the two tests can be helpful to generate appropriate estimates of these parameters. Furthermore, the true population prevalence was higher in the partial depletion population than in the final depletion population. This is in contrast to previous studies that demonstrated that Campylobacter colonization of a flock increased with time (Hald et al., 2000, 2001; Jacobs-Reitsma et al., 2001; Russa et al., 2005). The high prevalence in partial and the low prevalence in final depletion population might be because about $50 \%$ of the partial depletion populations and $90 \%$ of the final depletion flocks came from different flocks with distinct prevalence where the former was higher.

Interventions aimed at reducing the likelihood of exposure of consumers to Campylobacter directly from poultry and poultry products are expected to contribute to a reduced incidence of illness in humans. Our results may help in improving Campylobacter monitoring programmes to reduce the number of false negative flocks. Taking fecal samples has advantages, being easy to collect and less costly. The herd sensitivity of fecal culture could be increased by taking more samples, by improving the handling of samples between collection and laboratory testing (see for example Musgrove et al., 2001). Furthermore Campylobacter prevalence reports based on fecal culture of broilers should also consider the low sensitivity of the test, which may underestimate the true value. This is mainly important in risk analysis studies, which make use of prevalence and sensitivity of the test used. Further study could be carried out to get a better estimate with sufficiently large data and with controlled potential extraneous factors such as season and environment and the interval between collection of samples and laboratory testing that may have impact on the culturing process. Then, the surveillance system could be improved and necessary measures to reduce human exposure could possibly be developed.

\section{References}

Adkin, A., Hartnett, E., Jordan, L., Newell, D., Davison, H., 2006. Use of a systematic review to assist the development of Campylobacter control strategies in broilers. Journal of Applied Microbiology 100, 306-315.

Altekruse, S.F., Stern, N.J., Fields, P.I., Swerdlow, D.L., 1999. Campylobacter jejuni — an emerging foodborne pathogen. Emerging Infectious Diseases 5, $28-35$.

Berndston, E., Danielsen-Tham, D.L., Engvall, A., 1996. Campylobacter incidence on a chicken farm and the spread of Campylobacter during the slaughter process. International Journal of Food Microbiology 32, 35-47. 
Branscum, A.J., Gardner, I.A., Johnson, W.O., 2005. Estimation of diagnostictest sensitivity and specificity through Bayesian modelling. Preventive Veterinary Medicine 68, 145-163.

Casella, G., George, E., 1992. Explaining the Gibbs sampler. American Statistician 46, 167-174.

Corry, J.E.L., Post, D.E., Colin, P., Laisney, M.J., 1995. Culture media for the isolation of Campylobacters. International Journal of Food Microbiology 26, 43-76.

De Wit, M.A., Koopmans, M.P., Kortbeek, L.M., Van Leevwen, N.J., Bartelds, A.I., Duynhoven, Y.T., 2001. Gastroeneteritis in sentinel general practices, The Netherlands. Emerging Infectious Diseases 7, 82-91.

Dohoo, I., Martin, W., Stryhn, H., 2003. Veterinary Epidemiologic Research. AVC Inc., Charlottetown, Prince Edward Island, Canada.

Engberg, J., Aarestrup, F.M., Taylor, D.E., Gerner-Smidt, P., Nachamkin, I., 2001. Quinolone and macrolide resistance in Campylobacter jejuni and C. coli: resistance mechanisms and trends in human isolates. Emerging Infectious Diseases 7, 24-34.

Engel, B., Swildens, B., Stegeman, A., Buist, W., De Jong, M., 2006. Estimation of sensitivity and specificity of three conditionally dependent diagnostic tests in the absence of a gold standard. Journal of Agricultural Biological Environmental Statistics 11, 360-380.

Enøe, C., Georgiadis, M.P., Johnson, W.O., 2001. Estimation of sensitivity and specificity of diagnostic tests and disease prevalence. Preventive Veterinary Medicine 45, 61-81.

Frossling, J., Bonnett, B., Lindberg, A., Bjorkman, C., 2003. Validation of Neospora caninum iscom ELISA without a gold standard. Preventive Veterinary Medicine 57, 141-153.

Gardner, I.A., Stryhn, H., Lind, P., Collins, M.T., 2000. Conditional dependence between tests affects the diagnosis and surveillance of animal diseases. Preventive Veterinary Medicine; 45, 107-122.

Gelman, A., Rubin, D.B., 1992. Inference from iterative simulation using multiple sequences. Statistical Science 7, 457-472.

Georgiadis, M.P., Johnson, W.O., Gardner, I.A., Singh, R., 2003. Correlationadjusted estimation of sensitivity and specificity of two diagnostic tests. Applied Statistics 52, 63-76.

Hald, B., Wedderkopp, A., Madsen, M., 2000. Thermophilic Campylobacter spp. in Danish broiler production: a cross sectional survey and a retrospective analysis of risk factors for occurrence in broiler flocks. Avian Pathology 29, 123-131.

Hald, B., Rottenborg, E., Madsen, M., 2001. Role of batch depletion of broiler houses on the occurrence of Campylobacter spp. in chicken flocks. Letters in Applied Microbiology 32, 253-256.

Havaei, S.A., Salehi, R., Bokaeian, M., Fazeli, S.A., 2006. Comparison of PCR and culture methods for diagnosis of enteropathogenic Campylobacter in fowl feces. Iranian Biomedical Journal 10, 47-50.

Havelaar, A., 2004. CARMA: a multidisciplinary project to reduce risk of campylobacteriosis. Workshop on Prioritizing Opportunities to Reduce Foodborne Diseases, June 15-16. Iowa State University, Ames, IA.
Jacobs-Reitsma, W.F., 1997. Aspects of epidemiology of Campylobacter in poultry. Veterinary Quarterly 19, 113-117.

Jacobs-Reitsma, W., Wilpshaar, E., Gussinklo, B., Wagenaar, J., Stegeman, A., 2001. Epidemiological investigations into the colonization of Dutch broiler flocks with Campylobacter. The 11th International Workshop on Campylobacter, Helicobacter and Related Organisms (CHRO). International Journal of Medical Microbiology 291 (S31), 42-43.

Lund, M., Nordentoft, N., Pedersen, K., Madsen, M., 2004. Detection of Campylobacter spp. in chicken fecal samples by real-time PCR. Journal of Clinical Microbiology 42, 5125-5132.

Musgrove, M.J., Berrand, M.E., Byrd, J.A., Stern, N.J., Cox, N.A., 2001 Detection of Campylobacter spp in ceca and crops with and without enrichment. Poultry Science 80, 825-828.

Newell, D.G., Fearnley, C., 2003. Sources of Campylobacter colonization in broiler chickens. Applied and Environmental Microbiology 69, 4343-4351.

Orr, K.A., O'Reilly, K.L., Scholl, D.T., 2003. Estimation of sensitivity and specificity of two diagnostics tests for bovine immunodeficiency virus using Bayesian techniques. Preventive Veterinary Medicine 61, 79-89.

Payne, R.E., Margie, D.L., David, W.D., Harold, M.B., 1999. Molecular epidemiology of Campylobacter jejuni in broiler flocks using randomly amplified polymorphic DNA-PCR and 23 s RNA-PCR and role of litter in its transmission. Applied and Environmental Microbiology 65, 260-263.

Rudi, K., Høidal, H.K., Katla, T., Johansen, B.K., Nordal, J., Jakobsen, K.S., 2004. Direct real-time PCR quantification of Campylobacter jejuni in chicken fecal and cecal samples by integrated cell concentration and DNA purification. Applied and Environmental Microbiology 70, 790-797.

Russa, A.D., Bouma, A., Vernooij, J.C.M., Jacobs-Reitsma, W., Stegeman, J.A., 2005. No association between partial depopulation and Campylobacter spp. colonisation of Dutch broiler flocks. Letters in Applied Microbiology 41, 280-285.

Skirrow, M.B., 1991. Epidemiology of Campylobacter enteritis. International Journal of Food Microbiology 12, 9-16.

Spiegelhalter, D.J., Thomas, A., Best, N.G., Gilks, W.R., 1996. BUGS Bayesian inference Using Gibbs Sampling, Version 0.5. MRC Biostatistics Unit, Cambridge. http://www.mrc-bsu.cam.ac.uk/bugs/winbugs/contents. shtml.

Swildens, B., Wisselink, H.J., Engel, B., Smith, H.E., Nielen, M., Verheijden, J.H.M., Stegeman, J.A., 2005. Detection of extracellular factor-positive Streptococcus suis serotype 2 strains in tonsillar swabs of live sows by PCR. Veterinary Microbiology 109, 223-228.

Wagenaar, J.A., Mevius, D.J., Havelaar, A.H., 2006. Campylobacter in primary animal production and control strategies to reduce the burden of human campylobacteriosis. Revue scientifique et technique 25, 581-894.

Wingstrand, A., Neimann, J., Engbreg, J., Nielsen, E.M.P., Gerner- Smidt, H.C., Wegner, K., Molbak, 2006. Fresh chicken as main risk factor for campylobacteriosis, Denmark. Emerging Infectious Diseases 12, 280-284. 